\title{
First encounter of extrapulmonary-gastrointestinal manifestation of SARS-CoV- 2 in a CoV+ patient in Cantonal Hospital Zenica
}

\author{
Alma Kubat ${ }^{1}$, Ilma Dadić ${ }^{1}$, Amel Džanić ${ }^{1}$, Anhel Koluh ${ }^{2}$ \\ ${ }^{1}$ Faculty of Medicine, University of Sarajevo, Bosnia and Herzegovina \\ ${ }^{2}$ Department of Surgery, Cantonal Hospital Zenica, Bosnia and Herzegovina \\ DOI: https://doi.org/10.26800/LV-142-supp5-16
}

\section{Background:}

The Covid-19 pandemic has suddenly spread around the world and presented primarily with symptoms common for respiratory diseases. Over time, It has been noticed that Covid 19 patients increasingly develop serious extrapulmonary complications as we will show in our case.

\section{Case presentation:}

Our patient is 49 years old man who is presented with general symptoms of infection and positive PCR test for SARS-CoV-2. He was one of the first patients infected with the corona virus in B\&H who was primarily hospitalized due to exacerbation of his condition in form of Bilateral interstitial pneumonia. Ten days after hospitalization an $\mathrm{x}$-ray was done to evaluate abdominal pain and showed distended short bowel, air fluid levels and free fluid in the abdomen which indicated ileus. With all necessary measures for protection for Covid-19 infection, the emergency laparotomy was performed and jejunoileal anastomosis with a protective ileostomy was done. Even though his second PCR test was negative his condition continue to progress. The patient was found to have bowel infarction due to ischemia with patchy necrosis and fibrin thrombi in mesenteric arteries. He developed perforation of the gangrenosum parts of the small intestine, and underwent re-laparotomy and resection. He was prescribed adequate therapy to prevent additional complications.

\section{Conclusion:}

Patients with COVID-19 often develop gastrointestinal complications during their hospital stay, including bowel ischemia, gastrointestinal bleeding, and severe ileus. What may seem like a Covid 19 infection must not be treated just as a respiratory infection, but with a multidisciplinary approach which is crucial for a successful final outcome.

Keywords: Covid-19, gastrointestinal manifestation, laparotomy 